\title{
ON THE DISTRIBUTIONS OF A RENEWAL REWARD PROCESS AND IT'S ADDITIVE FUNCTIONAL
}

\author{
Tahir Khaniyevev,c, ${ }^{\mathrm{a}, \text { }}$ Rovshan Aliyev ${ }^{\mathrm{a}, \mathrm{c}}$, Zafer Küçük ${ }^{\mathrm{a}}$ and Nurgul Okur Bekar ${ }^{\mathrm{b}}$ \\ ${ }^{a}$ Karadeniz Technical University, Faculty of Arts and Sciences \\ Department of Statistics and Computer Sciences, 61080, Trabzon, Turkey \\ ${ }^{\mathrm{b}}$ Karadeniz Technical University, Faculty of Arts and Sciences, \\ Department of Mathematics, 61080, Trabzon, Turkey \\ ${ }^{\mathrm{c}}$ Institute of Cybernetics of Azerbaijan National Academy of Sciences, \\ F. Agayev str.9, Az 1141, Baku, Azerbaijan \\ e-mail: khaniyevtahir@gmail.com
}

\begin{abstract}
In this study, a renewal reward process with a discrete interference of chance $(\mathrm{X}(\mathrm{t}))$ is constructed and distribution of the process $\mathrm{X}(\mathrm{t})$ is investigated. Onedimensional distribution of the process $\mathrm{X}(\mathrm{t})$ is given by means of the probability characteristics of the renewal processes $\left\{T_{n}\right\}$ and $\left\{S_{n}\right\}$. Moreover, one dimensional distribution function of the additive functional $J_{f}(t)$ of the process $X(t)$ is expressed by the probability characteristics of the initial sequences of the random variables $\left\{\xi_{n}\right\}$ and $\left\{\eta_{n}\right\}$.
\end{abstract}

Keywords- Renewal Reward Process, Additive Functional, Finite Dimensional Distribution, Discrete Interference of Chance.

\section{INTRODUCTION}

A number of very interesting problems of queuing, reliability, risk, sequential analysis and control of reserves theories, mathematical insurance, statistics, biology and physics are expressed by means of the renewal reward process with a discrete interference of chance. Numereous studies have been done about a renewal reward process with a discrete interference of chance because of their practical and theoretical importance. Jewell [4] generalized the study of the fluctuations of a reward process imbedded in a renewal process. Brown and Solomon [2] obtained second order asymptotic expansions for the first and second moments of the renewal reward process. Khaniyev [10] investigated a generalized renewal process $\mathrm{T}_{\mathrm{N}(\mathrm{x})}=\sum_{\mathrm{i}=1}^{\mathrm{N}(\mathrm{x})} \xi_{\mathrm{i}}$, where $N(x)=\inf \left\{n \geq 1: T_{n}>x\right\}$ and $\left\{\xi_{i}\right\}, i \geq 1$, is a sequence of independent and identically distributed positive random variables. Khaniyev obtained analytic results about moments of the process. Then, in this study an asymtpotic behavior, as $t \rightarrow \infty$ of the process is investigated.

Morever, a number of authors have investigated the harmonic renewal measure $\mathrm{U}_{1}\{\mathrm{I}\}=\sum_{\mathrm{n}=1}^{\infty} 1 / \mathrm{n} \mathrm{P}\left\{\mathrm{S}_{\mathrm{n}} \in \mathrm{I}\right\}, \mathrm{I} \subseteq \mathrm{R}$. We mention here contributions by Alsmeyer [1]. It is 
not difficult to see that there exist some connection between harmonic renewal measures and first passage times. For instance, Alsmeyer proved that if $E\left(\eta_{1}\right) \equiv \mu>0$ then the following asymptotic expansion is true as $\mathrm{x} \rightarrow \infty$ :

$\mathrm{U}_{1}(\mathrm{x})=\log (\mathrm{x} / \mu)+\gamma+\mathrm{o}(1)$,

where $\mathrm{U}_{1}(\mathrm{x})=\sum_{\mathrm{n}=1}^{\infty} 1 / \mathrm{nP}\left\{\mathrm{S}_{\mathrm{n}} \leq \mathrm{x}\right\}$ is a harmonic renewal function, $\mathrm{S}_{\mathrm{n}}=\sum_{\mathrm{i}=1}^{\mathrm{n}} \eta_{\mathrm{i}}, \mathrm{n} \geq 1$, $\left\{\eta_{\mathrm{n}}\right\}$ is a sequence of independent and identically distributed random variables and $\gamma$ is Euler's constant. But the probability characteristics of additive functional of the renewal reward process aren't sufficient investigate in literature, which is very important for solving some problems of applied sciences.

Therefore, in this study, a renewal reward process with a discrete interference of chance $(X(t))$ and it's additive functional $\left(J_{f}(t)\right)$ are constructed mathematically and the one dimensional distribution functions of the process $\mathrm{X}(\mathrm{t})$ and additive function $\mathrm{J}_{\mathrm{f}}(\mathrm{t})$ are calculated.

\section{CONSTRUCTION OF THE PROCESS AND IT'S ADDITIVE FUNCTIONAL}

Let $\left\{\left(\xi_{n}, \eta_{n}, \zeta_{n}\right)\right\}, n \geq 1$, be a sequence of independent and identically distributed triples of random variables, defined on any probability space $(\Omega, \mathfrak{I}, \mathrm{P})$, where $\xi_{\mathrm{n}}$ 's and $\eta_{\mathrm{n}}$ 's take only positive values; $\zeta_{\mathrm{n}}$ 's take values from interval $[\mathrm{s},+\infty)$, where $\mathrm{s}$ is any real positive number. Suppose that $\xi_{1}, \eta_{1}, \zeta_{1}$ are mutually independent random variables and let's denote the distribution of $\xi_{1}, \eta_{1}$ and $\zeta_{1}$ by $\Phi(t), F(x)$ and $\pi(z)$, respectively, i.e.,

$$
\Phi(\mathrm{t})=\mathrm{P}\left\{\xi_{1} \leq \mathrm{t}\right\}, \mathrm{F}(\mathrm{x})=\mathrm{P}\left\{\eta_{1} \leq \mathrm{x}\right\}, \pi(\mathrm{z})=\mathrm{P}\left\{\zeta_{1} \leq \mathrm{z}\right\}, \mathrm{t}, \mathrm{x} \geq 0, \mathrm{z} \geq \mathrm{s} .
$$

Define the renewal sequnces $\left\{T_{n}\right\},\left\{S_{n}\right\}$ by using the random pairs $\left(\xi_{i}, \eta_{i}\right), i \geq 1$ :

$$
\mathrm{T}_{\mathrm{n}}=\sum_{\mathrm{i}=1}^{\mathrm{n}} \xi_{\mathrm{i}}, \mathrm{S}_{\mathrm{n}}=\sum_{\mathrm{i}=1}^{\mathrm{n}} \eta_{\mathrm{i}}, \mathrm{n} \geq 1, \mathrm{~T}_{0}=\mathrm{S}_{0}=0,
$$

and a sequence of integer valued random variables $\mathrm{N}_{\mathrm{n}}$ as:

$$
\mathrm{N}_{0}=0 ; \quad \mathrm{N}_{\mathrm{n}+1}=\inf \left\{\mathrm{k} \geq \mathrm{N}_{\mathrm{n}}+1: \zeta_{\mathrm{n}}-\mathrm{S}_{\mathrm{k}}+\mathrm{S}_{\mathrm{N}_{\mathrm{n}}}<\mathrm{s}\right\}, \mathrm{n} \geq 0
$$

where $\inf (\varnothing)=+\infty$ is stipulated. Moreover, put

$$
\tau_{\mathrm{n}}=\mathrm{T}_{\mathrm{N}_{\mathrm{n}}}=\sum_{\mathrm{i}=1}^{\mathrm{N}_{\mathrm{n}}} \xi_{\mathrm{i}} ; \mathrm{n} \geq 1, \tau_{0}=0 ; \mathrm{v}(\mathrm{t})=\max \left\{\mathrm{n} \geq 0: \mathrm{T}_{\mathrm{n}} \leq \mathrm{t}\right\}, \mathrm{t}>0 .
$$

We can now construct desired stochastic process $\mathrm{X}(\mathrm{t})$ as follows:

$$
X(t)=\max \left\{s, \zeta_{n}-S_{v(t)}+S_{N_{n}}\right\}, \text { for each } t \in\left[\tau_{n}, \tau_{n+1}\right), n \geq 0,
$$

where $\zeta_{0}=\mathrm{z} \in[\mathrm{s},+\infty)$ and $\mathrm{S}_{v\left(\tau_{\mathrm{n}}+0\right)}=\mathrm{S}_{\mathrm{N}_{\mathrm{n}}}$.

The process $\mathrm{X}(\mathrm{t})$ is called a renewal reward process with a discrete interference of chance. The following graph is one of the trajectories of the process $X(t)$ : 


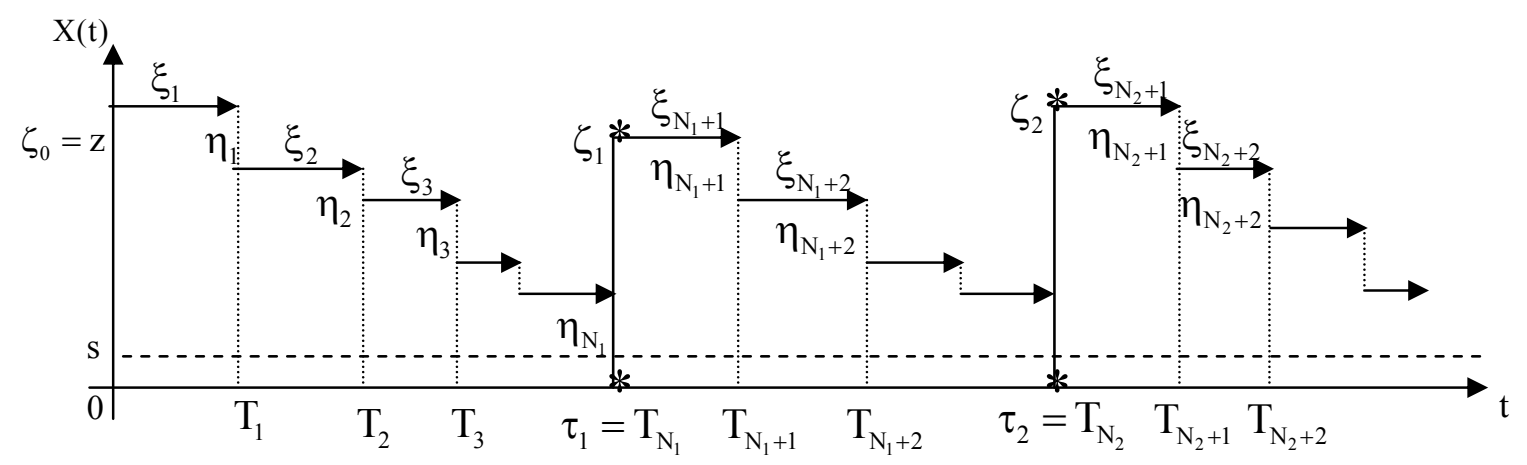

Picture 1. One of the trajectories of the process $X(t)$

Let $\mathrm{f}: \mathrm{R} \rightarrow \mathrm{R}$ is a bounded measurable function. For each $\mathrm{t} \geq 0$, define $\mathrm{J}_{\mathrm{f}}(\mathrm{t})$ as follows: $J_{f}(t)=\int_{0}^{t} f(X(u)) d u$. $J_{f}(t)$ is called an additive functional of the process $X(t)$. The main purpose of this study is to investigate the distributions of the renewal reward process $\mathrm{X}(\mathrm{t})$ and it's additive functional $\mathrm{J}_{\mathrm{f}}(\mathrm{t})$.

\section{ONE DIMENSIONAL DISTRIBUTION FUNCTIONS OF THE PROCESS X(t)}

Let us denote by $\mathrm{Q}(\mathrm{t}, \mathrm{x}, \mathrm{z})$ one dimensional distrubition function of the process $\mathrm{X}(\mathrm{t})$, i.e., $\mathrm{Q}(\mathrm{t}, \mathrm{x}, \mathrm{z}) \equiv \mathrm{P}_{\mathrm{z}}\{\mathrm{X}(\mathrm{t}) \leq \mathrm{X}\} \equiv \mathrm{P}\{\mathrm{X}(\mathrm{t}) \leq \mathrm{X} \mid \mathrm{X}(0)=\mathrm{z}\}, \mathrm{t}, \mathrm{X} \geq 0, \mathrm{z} \geq \mathrm{s}$.

The main purpose of this study is to express one dimensional distrubition function $\mathrm{Q}(\mathrm{t}, \mathrm{x}, \mathrm{z})$ of the process $\mathrm{X}(\mathrm{t})$ by means of probability characteristics of the initial sequence of random variables $\left\{\left(\xi_{n}, \eta_{n}, \zeta_{n}\right)\right\}, n \geq 1$. Define Laplace transform and Laplace-Stiltijes transform of the bounded measurable function $\mathrm{M}(\mathrm{t}, \mathrm{x}, \mathrm{z})$ as follows:

$$
\tilde{M}(\lambda, x, z)=\int_{0}^{\infty} e^{-\lambda t} M(t, x, z) d t ; M^{*}(\lambda, x, z)=\int_{0}^{\infty} e^{-\lambda t} d_{t} M(t, x, z)
$$

In order to becoming more precise now we need further notation first:

$$
\begin{aligned}
& \Phi_{0}(\mathrm{t})=\Phi^{* 0}(\mathrm{t})=\varepsilon(\mathrm{t})=\left\{\begin{array}{ll}
1, & \mathrm{t} \geq 0 \\
0, & \mathrm{t}<0
\end{array}, \quad \Phi_{\mathrm{n}}(\mathrm{t})=\mathrm{P}\left\{\mathrm{T}_{\mathrm{n}} \leq \mathrm{t}\right\}=\Phi^{*_{\mathrm{n}}}(\mathrm{t}), \mathrm{n} \geq 1,\right. \\
& \mathrm{F}_{0}(\mathrm{x})=\varepsilon(\mathrm{x}), \mathrm{F}_{\mathrm{n}}(\mathrm{x})=\mathrm{P}\left\{\mathrm{S}_{\mathrm{n}} \leq \mathrm{x}\right\}, \mathrm{n} \geq 1, \mathrm{x}=\mathrm{z}-\mathrm{s}, \mathrm{z} \geq \mathrm{s}, \\
& \mathrm{M}(\mathrm{t}, \mathrm{x}, \bullet)=\int_{\mathrm{s}}^{+\infty} \mathrm{M}(\mathrm{t}, \mathrm{x}, \mathrm{z}) \mathrm{d} \pi(\mathrm{z}), \mathrm{a}_{\mathrm{n}}(\mathrm{x}, \mathrm{z})=\mathrm{F}_{\mathrm{n}}(\mathrm{z}-\mathrm{s})-\mathrm{F}_{\mathrm{n}}(\mathrm{z}-\mathrm{x}), \mathrm{x} \in[\mathrm{s}, \mathrm{z}], \\
& \mathrm{G}(\mathrm{t}, \mathrm{x}, \mathrm{z})=\sum_{\mathrm{n}=0}^{\infty} \Delta \Phi_{\mathrm{n}}(\mathrm{t}) \mathrm{a}_{\mathrm{n}}(\mathrm{x}, \mathrm{z}) ; \Delta \Phi_{\mathrm{n}}(\mathrm{t})=\Phi_{\mathrm{n}}(\mathrm{t})-\Phi_{\mathrm{n}+1}(\mathrm{t}), \mathrm{n} \geq 0,
\end{aligned}
$$




$$
\mathrm{R}(\mathrm{t}, \mathrm{z})=\sum_{\mathrm{n}=1}^{\infty} \Phi_{\mathrm{n}}(\mathrm{t}) \mathrm{b}_{\mathrm{n}}(\mathrm{z}-\mathrm{s}) ; \mathrm{b}_{\mathrm{n}}(\mathrm{z}-\mathrm{s})=\mathrm{F}_{\mathrm{n}-1}(\mathrm{z}-\mathrm{s})-\mathrm{F}_{\mathrm{n}}(\mathrm{z}-\mathrm{s})
$$

Theorem 3.1. If $\xi_{1}, \eta_{1}$ and $\zeta_{1}$ are independent, then the Laplace transform of the distrubition functions $\mathrm{Q}(\mathrm{t}, \mathrm{X}, \mathrm{z})$ of the process $\mathrm{X}(\mathrm{t})$ can be expressed by the probability characteristics of the initial random variables, as follows:

$$
\widetilde{\mathrm{Q}}(\lambda, \mathrm{x}, \mathrm{z})=\widetilde{\mathrm{G}}(\lambda, \mathrm{x}, \mathrm{z})+\frac{\mathrm{R}^{*}(\lambda, \mathrm{z}) \widetilde{\mathrm{G}}(\lambda, \mathrm{x}, \bullet)}{1-\mathrm{R}^{*}(\lambda, \bullet)} .
$$

Proof. Using total probability formula, the one dimensional distrubition function $\mathrm{Q}(\mathrm{t}, \mathrm{X}, \mathrm{Z})$ of the process $\mathrm{X}(\mathrm{t})$ can be written as follows:

$$
\mathrm{Q}(\mathrm{t}, \mathrm{x}, \mathrm{z})=\mathrm{P}_{\mathrm{z}}\{\mathrm{X}(\mathrm{t}) \leq \mathrm{x}\}=\mathrm{P}_{\mathrm{z}}\left\{\mathrm{t}<\tau_{1} ; \mathrm{X}(\mathrm{t}) \leq \mathrm{x}\right\}+\mathrm{P}_{\mathrm{z}}\left\{\mathrm{t} \geq \tau_{1} ; \mathrm{X}(\mathrm{t}) \leq \mathrm{x}\right\} .
$$

Let the first term of the equality (3.3) be $\mathrm{G}(\mathrm{t}, \mathrm{x}, \mathrm{z})$. Let's rewrite the second term of the equality (3.3) as follows:

$$
\begin{aligned}
\mathrm{P}_{\mathrm{z}}\left\{\mathrm{t} \geq \tau_{1} ; \mathrm{X}(\mathrm{t}) \leq \mathrm{x}\right\} & =\int_{0}^{\mathrm{t}} \mathrm{P}_{\mathrm{z}}\left\{\tau_{1} \in \mathrm{du} ; \mathrm{X}(\mathrm{t}) \leq \mathrm{x}\right\} \\
& =\int_{0}^{\mathrm{t}} \int_{\mathrm{s}}^{+\infty} \mathrm{Q}(\mathrm{t}-\mathrm{u}, \mathrm{x}, \mathrm{v}) \mathrm{R}(\mathrm{du}, \mathrm{z}) \pi\{\mathrm{dv}\} \\
& =\int_{0}^{\mathrm{t}} \mathrm{R}(\mathrm{du}, \mathrm{z}) \int_{\mathrm{s}}^{+\infty} \mathrm{Q}(\mathrm{t}-\mathrm{u}, \mathrm{x}, \mathrm{v}) \pi\{\mathrm{dv}\} \\
& =\int_{0}^{\mathrm{t}} \mathrm{R}(\mathrm{du}, \mathrm{z}) \mathrm{Q}(\mathrm{t}-\mathrm{u}, \mathrm{x}, \bullet)=\mathrm{R}(\mathrm{t}, \mathrm{z}) * \mathrm{Q}(\mathrm{t}, \mathrm{x}, \bullet) .
\end{aligned}
$$

Therefore, the equality (3.3) can be written as follows:

$$
\mathrm{Q}(\mathrm{t}, \mathrm{x}, \mathrm{z})=\mathrm{G}(\mathrm{t}, \mathrm{x}, \mathrm{z})+\mathrm{R}(\mathrm{t}, \mathrm{z}) * \mathrm{Q}(\mathrm{t}, \mathrm{x}, \bullet) \text {. }
$$

If both sides of the the equality (3.4) are multiplied by $\pi(\mathrm{dz})$ and integrated respect to parameter $\mathrm{z}$ from $\mathrm{s}$ to $+\infty$, we get:

$$
\mathrm{Q}(\mathrm{t}, \mathrm{x}, \bullet)=\mathrm{G}(\mathrm{t}, \mathrm{x}, \bullet)+\mathrm{R}(\mathrm{t}, \bullet) * \mathrm{Q}(\mathrm{t}, \mathrm{x}, \bullet) \text {. }
$$

Moreover, if both sides of the equality (3.5) are multiplied by $\mathrm{e}^{-\lambda \mathrm{t}}$ and integrated respect to parameter $t$, we get:

$$
\widetilde{\mathrm{Q}}(\lambda, \mathrm{x}, \bullet)=\widetilde{\mathrm{G}}(\lambda, \mathrm{x}, \bullet)+\mathrm{R}^{*}(\lambda, \bullet) \widetilde{\mathrm{Q}}(\lambda, \mathrm{x}, \bullet) .
$$

Then, we can write:

$$
\widetilde{\mathrm{Q}}(\lambda, \mathrm{x}, \bullet)=\frac{\widetilde{\mathrm{G}}(\lambda, \mathrm{x}, \bullet)}{1-\mathrm{R}^{*}(\lambda, \bullet)} .
$$


Using the formulas (3.6) and (3.7), we can write:

$$
\widetilde{\mathrm{Q}}(\lambda, \mathrm{x}, \mathrm{z})=\widetilde{\mathrm{G}}(\lambda, \mathrm{x}, \mathrm{z})+\frac{\mathrm{R}^{*}(\lambda, \mathrm{z}) \widetilde{\mathrm{G}}(\lambda, \mathrm{x}, \bullet)}{1-\mathrm{R}^{*}(\lambda, \bullet)} .
$$

The above result show that $\widetilde{Q}(\lambda, x, z)$ can be expressed using $\widetilde{G}(\lambda, x, z)$ and $R^{*}(\lambda, x, z)$.

Let's now express functions $G(t, x, z)$ and $R(t, x, z)$ by the certain probability characteristics of the initial sequence of random variables.

$$
\begin{aligned}
\mathrm{G}(\mathrm{t}, \mathrm{x}, \mathrm{z})=\mathrm{P}_{\mathrm{z}}\left\{\tau_{1}>\mathrm{t} ; \mathrm{X}(\mathrm{t}) \leq \mathrm{x}\right\}=\sum_{\mathrm{n}=0}^{\infty} \mathrm{P}_{\mathrm{z}}\left\{\mathrm{v}(\mathrm{t})=\mathrm{n} ; \tau_{1}>\mathrm{t}, \mathrm{X}(\mathrm{t}) \leq \mathrm{x}\right\} \\
=\sum_{\mathrm{n}=0}^{\infty} \mathrm{P}\left\{\mathrm{T}_{\mathrm{n}}<\mathrm{t}<\mathrm{T}_{\mathrm{n}+1} ; \mathrm{z}-\mathrm{S}_{\mathrm{n}}>\mathrm{s} ; \mathrm{z}-\mathrm{S}_{\mathrm{n}} \leq \mathrm{x}\right\}=\sum_{\mathrm{n}=0}^{\infty} \Delta \Phi_{\mathrm{n}}(\mathrm{t}) \mathrm{a}_{\mathrm{n}}(\mathrm{x}, \mathrm{z}) .
\end{aligned}
$$

Let's now calculate $\mathrm{R}(\mathrm{du}, \mathrm{z})$ :

$$
\mathrm{R}(\mathrm{du}, \mathrm{z})=\mathrm{P}_{\mathrm{z}}\left\{\mathrm{T}_{\mathrm{N}_{1}} \in \mathrm{du}\right\}=\sum_{\mathrm{n}=1}^{\infty} \mathrm{P}_{\mathrm{z}}\left\{\mathrm{N}_{1}=\mathrm{n} ; \mathrm{T}_{\mathrm{n}} \in \mathrm{du}\right\}=\sum_{\mathrm{n}=1}^{\infty} \mathrm{b}_{\mathrm{n}}(\mathrm{z}-\mathrm{s}) \mathrm{d} \Phi_{\mathrm{n}}(\mathrm{u})
$$

Therefore, we finally get:

$$
\mathrm{R}(\mathrm{t}, \mathrm{z})=\mathrm{P}_{\mathrm{z}}\left\{\tau_{1} \leq \mathrm{t}\right\}=\sum_{\mathrm{n}=1}^{\infty} \Phi_{\mathrm{n}}(\mathrm{t}) \mathrm{b}_{\mathrm{n}}(\mathrm{z}-\mathrm{s})
$$

This completes the proof of the Theorem 3.1.

\section{ONE DIMENSIONAL DISTRIBUTION FUNCTION OF THE ADDITIVE FUNCTIONAL $\mathbf{J}_{\mathrm{f}}(\mathbf{t})$}

Let us denote by $\mathrm{Q}_{\mathrm{f}}(\mathrm{t}, \mathrm{x}, \mathrm{z})$, the one dimensional distrubition function of the additive functional $\mathrm{J}_{\mathrm{f}}(\mathrm{t})$, i.e., $\mathrm{Q}_{\mathrm{f}}(\mathrm{t}, \mathrm{x}, \mathrm{z})=\mathrm{P}_{\mathrm{z}}\left\{\mathrm{J}_{\mathrm{f}}(\mathrm{t}) \leq \mathrm{x}\right\}, \mathrm{t}, \mathrm{x} \geq 0, \mathrm{z} \geq \mathrm{s}$.

In order to becoming more precise now we need further notation first.

$\mathrm{G}_{\mathrm{f}}(\mathrm{t}, \mathrm{x}, \mathrm{z})=$

$$
\begin{aligned}
& =\sum_{\mathrm{n}=0}^{\infty} \mathrm{P}\left\{\mathrm{T}_{\mathrm{n}} \leq \mathrm{t}<\mathrm{T}_{\mathrm{n}+1} ; \mathrm{z}-\mathrm{S}_{\mathrm{n}}>\mathrm{s} ; \sum_{\mathrm{k}=0}^{\mathrm{n}-1} \mathrm{f}\left(\mathrm{z}-\mathrm{S}_{\mathrm{k}}\right) \xi_{\mathrm{k}+1}+\left(\mathrm{t}-\mathrm{T}_{\mathrm{n}}\right) \mathrm{f}\left(\mathrm{z}-\mathrm{S}_{\mathrm{k}}\right) \leq \mathrm{x}\right\}, \\
& \mathrm{R}_{\mathrm{f}}(\mathrm{t}, \mathrm{x}, \mathrm{z})=\sum_{\mathrm{n}=1}^{\infty} \mathrm{P}\left\{\mathrm{T}_{\mathrm{n}} \leq \mathrm{t} ; \mathrm{z}-\mathrm{S}_{\mathrm{n}}<\mathrm{s}<\mathrm{z}-\mathrm{S}_{\mathrm{n}-1} ; \sum_{\mathrm{k}=0}^{\mathrm{n}-1} \mathrm{f}\left(\mathrm{z}-\mathrm{S}_{\mathrm{k}}\right) \xi_{\mathrm{k}+1} \leq \mathrm{x}\right\} .
\end{aligned}
$$

For each the bounded measurable function $\mathrm{M}(\mathrm{t}, \mathrm{x}, \mathrm{z})$, we define the following double transforms:

$$
\widetilde{\widetilde{M}}(\lambda, \mu, z)=\int_{0}^{\infty} \int_{-\infty}^{+\infty} \mathrm{e}^{-\lambda t} \mathrm{e}^{\mathrm{i} \mu \mathrm{x}} \mathrm{M}(\mathrm{t}, \mathrm{x}, \mathrm{z}) \mathrm{dtdx} ; \mathrm{M}^{* *}(\lambda, \mu, \mathrm{z})=\int_{0}^{\infty} \int_{-\infty}^{+\infty} \mathrm{e}^{-\lambda \mathrm{t}} \mathrm{e}^{\mathrm{i} \mu \mathrm{x}} \mathrm{M}(\mathrm{dt}, \mathrm{dx}, \mathrm{z}),
$$

where $\lambda>0, \mu \in \mathrm{R}$. 
Theorem 4.1. Suppose that $\xi_{1}$ and $\eta_{1}$ are independent random variables. Then the double transform of the one dimensional distribution function of the additive functional $\mathrm{J}_{\mathrm{f}}(\mathrm{t})$ can be written as follows:

$$
\widetilde{\widetilde{Q}}(\lambda, \mu, \mathrm{z})=\widetilde{\widetilde{G}}_{\mathrm{f}}(\lambda, \mu, \mathrm{z})+\widetilde{\widetilde{G}}_{\mathrm{f}}(\lambda, \mu, \bullet) \mathrm{R}_{\mathrm{f}}^{* *}(\lambda, \mu, \mathrm{z})\left[1-\mathrm{R}_{\mathrm{f}}^{* *}(\lambda, \mu, \bullet)\right]^{-1},
$$

where $G_{f}(t, x, z), \quad R_{f}(t, x, z)$ are expressed by the probability characteristics of sequences of the random variables $\left\{\xi_{n}\right\},\left\{\eta_{n}\right\}$ as the formulas (4.1) and (4.2).

Proof. Let's try to write obtained the integral equation of renewal reward type for $\mathrm{Q}_{\mathrm{f}}(\mathrm{t}, \mathrm{x}, \mathrm{z})$ :

$$
\mathrm{Q}_{\mathrm{f}}(\mathrm{t}, \mathrm{x}, \mathrm{z})=\mathrm{P}_{\mathrm{z}}\left\{\mathrm{J}_{\mathrm{f}}(\mathrm{t}) \leq \mathrm{x}\right\}=\mathrm{P}_{\mathrm{z}}\left\{\tau_{1}>\mathrm{t} ; \mathrm{J}_{\mathrm{f}}(\mathrm{t}) \leq \mathrm{x}\right\}+\mathrm{P}_{\mathrm{z}}\left\{\tau_{1} \leq \mathrm{t} ; \mathrm{J}_{\mathrm{f}}(\mathrm{t}) \leq \mathrm{x}\right\} .
$$

Denote the first term of the $(4.3)$ by $G_{f}(t, x, z)$. Let's rewritte the second term of the equation (4.3) as follows:

$$
\begin{aligned}
& P_{z}\left\{\tau_{1} \leq t ; J_{f}(t) \leq x\right\}= \\
& =\int_{0}^{t} \int_{s}^{+\infty+\infty} \int_{-\infty} P_{z}\left\{\tau_{1} \in d u ; X\left(\tau_{1}\right) \in d v ; J_{f}\left(\tau_{1}\right) \in d y\right\} P_{v}\left\{J_{f}(t-u) \leq x-y\right\} \\
& =\int_{0}^{t} \int_{s}^{+\infty} \int_{-\infty}^{\infty+\infty} R_{f}(d u, d y, z) d \pi(v) Q(t-u, x-y, v),
\end{aligned}
$$

where

$$
\mathrm{d} \pi(\mathrm{v})=\mathrm{P}\left\{\zeta_{1} \in \mathrm{dv}\right\}, \mathrm{R}_{\mathrm{f}}(\mathrm{du}, \mathrm{dy}, \mathrm{z})=\mathrm{P}_{\mathrm{z}}\left\{\tau_{1} \in \mathrm{du} ; \mathrm{J}_{\mathrm{f}}\left(\tau_{1}\right) \in \mathrm{dy}\right\} .
$$

Substituting the (4.4) in the (4.3) we can obtain the integral equation as follows:

$$
\mathrm{Q}_{\mathrm{f}}(\mathrm{t}, \mathrm{x}, \mathrm{z})=\mathrm{G}_{\mathrm{f}}(\mathrm{t}, \mathrm{x}, \mathrm{z})+\int_{0}^{\mathrm{t}} \int_{\mathrm{s}}^{+\infty} \int_{-\infty}^{+\infty} \mathrm{R}_{\mathrm{f}}(\mathrm{du}, \mathrm{dy}, \mathrm{z}) \mathrm{Q}(\mathrm{t}-\mathrm{u}, \mathrm{x}-\mathrm{y}, \mathrm{v}) \mathrm{d} \pi(\mathrm{v}) .
$$

We can rewrite the equality (4.5) as follows:

$$
\mathrm{Q}_{\mathrm{f}}(\mathrm{t}, \mathrm{x}, \mathrm{z})=\mathrm{G}_{\mathrm{f}}(\mathrm{t}, \mathrm{x}, \mathrm{z})+\int_{0}^{\mathrm{t}} \int_{-\infty}^{+\infty} \mathrm{R}_{\mathrm{f}}(\mathrm{du}, \mathrm{dy}, \mathrm{z}) \mathrm{Q}(\mathrm{t}-\mathrm{u}, \mathrm{x}-\mathrm{y}, \bullet)
$$

The equation (4.6) is a integral equation of renewal reward type for $\mathrm{Q}_{\mathrm{f}}(\mathrm{t}, \mathrm{x}, \mathrm{z})$.

If both sides of the formula (4.6) are multiplied by $\mathrm{e}^{-\lambda t} \mathrm{e}^{-\mathrm{i} \mu x}$ and integrated respect to parameters $\mathrm{t}$, $\mathrm{x}$, we get:

$$
\widetilde{\widetilde{Q}}_{\mathrm{f}}(\lambda, \mu, \mathrm{z})=\widetilde{\widetilde{\mathrm{G}}}_{\mathrm{f}}(\lambda, \mu, \mathrm{z})+\widetilde{\widetilde{Q}}_{\mathrm{f}}(\lambda, \mu, \bullet) \mathrm{R}_{\mathrm{f}}^{* *}(\lambda, \mu, \mathrm{z}) .
$$

Averaging this equality respect to $\mathrm{z}$, we can obtained:

$$
\widetilde{\widetilde{Q}}_{\mathrm{f}}(\lambda, \mu, \bullet)=\widetilde{\widetilde{G}}_{\mathrm{f}}(\lambda, \mu, \bullet)+\widetilde{\mathrm{Q}}_{\mathrm{f}}(\lambda, \mu, \bullet) \mathrm{R}_{\mathrm{f}}^{* *}(\lambda, \mu, \bullet) .
$$


Then, we can write:

$$
\widetilde{\widetilde{Q}}(\lambda, \mu, \bullet)=\widetilde{\widetilde{G}}_{\mathrm{f}}(\lambda, \mu, \bullet)\left[1-\mathrm{R}_{\mathrm{f}}^{* *}(\lambda, \mu, \bullet)\right]^{-1} .
$$

Using the formulas (4.7) and (4.8) we can obtain as follows:

$$
\tilde{\tilde{Q}}_{\mathrm{f}}(\lambda, \mu, \mathrm{z})=\tilde{\tilde{\mathrm{G}}}_{\mathrm{f}}(\lambda, \mu, \mathrm{z})+\tilde{\tilde{\mathrm{G}}}_{\mathrm{f}}(\lambda, \mu, \bullet) \mathrm{R}_{\mathrm{f}}^{* *}(\lambda, \mu, \mathrm{z})\left[1-\mathrm{R}_{\mathrm{f}}^{* *}(\lambda, \mu, \bullet)\right]^{-1} .
$$

The above result show that the demanded function $\widetilde{\widetilde{Q}}_{\mathrm{f}}(\lambda, \mu, \mathrm{z})$ can be expressed by using $\widetilde{\widetilde{G}}_{\mathrm{f}}(\lambda, \mu, \mathrm{z})$ and $\mathrm{R}_{\mathrm{f}}^{* *}(\lambda, \mu, \mathrm{z}) . \mathrm{G}_{\mathrm{f}}(\mathrm{t}, \mathrm{x}, \mathrm{z})$ and $\mathrm{R}_{\mathrm{f}}(\mathrm{t}, \mathrm{x}, \mathrm{z})$ can be expressed by the certain probability characteristics of the initial sequence of random variables as follows:

$$
\begin{aligned}
& \mathrm{G}_{\mathrm{f}}(\mathrm{t}, \mathrm{x}, \mathrm{z}) \equiv \mathrm{P}_{\mathrm{z}}\left\{\tau_{1}>\mathrm{t}, \mathrm{J}_{\mathrm{f}}(\mathrm{t}) \leq \mathrm{x}\right\} \\
& =\sum_{\mathrm{n}=0}^{\infty} \mathrm{P}_{\mathrm{z}}\left\{\mathrm{v}(\mathrm{t})=\mathrm{n} ; v_{1}>\mathrm{n}+1 ; \int_{0}^{\mathrm{t}} \mathrm{f}(\mathrm{X}(\mathrm{u})) \mathrm{du} \leq \mathrm{x}\right\} \\
& =\sum_{\mathrm{n}=0}^{\infty} \mathrm{P}\left\{\mathrm{T}_{\mathrm{n}} \leq \mathrm{t}<\mathrm{T}_{\mathrm{n}+1} ; \mathrm{z}-\mathrm{S}_{\mathrm{n}}>\mathrm{s} ; \sum_{\mathrm{k}=0}^{\mathrm{n}-1} \mathrm{f}\left(\mathrm{z}+\mathrm{Y}_{\mathrm{k}}\right) \xi_{\mathrm{k}+1}+\left(\mathrm{t}-\mathrm{T}_{\mathrm{n}}\right) \mathrm{f}\left(\mathrm{z}-\mathrm{S}_{\mathrm{n}}\right) \leq \mathrm{x}\right\} ; \\
& \quad=\sum_{\mathrm{n}=1}^{\infty} \mathrm{P}\left\{\mathrm{T}_{\mathrm{n}} \in \mathrm{du} ; \mathrm{z}-\mathrm{S}_{\mathrm{n}}<\mathrm{s}<\mathrm{z}-\mathrm{S}_{\mathrm{n}-1} ; \sum_{\mathrm{k}=0}^{\mathrm{n}-1} \mathrm{f}\left(\mathrm{z}-\mathrm{S}_{\mathrm{k}}\right) \xi_{\mathrm{k}+1} \in \mathrm{dy}\right\} ; \\
& \mathrm{R}_{\mathrm{f}}(\mathrm{t}, \mathrm{dy}, \mathrm{z})=\sum_{\mathrm{n}=1}^{\infty} \mathrm{P}\left\{\mathrm{T}_{\mathrm{n}} \in \mathrm{du} ; \mathrm{z}-\mathrm{S}_{\mathrm{n}}<\mathrm{s}<\mathrm{z}-\mathrm{S}_{\mathrm{n}-1} ; \sum_{\mathrm{k}=0}^{\mathrm{n}-1} \mathrm{f}\left(\mathrm{z}-\mathrm{S}_{\mathrm{k}}\right) \xi_{\mathrm{k}+1} \in \mathrm{du} ; \mathrm{J}_{\mathrm{f}}\left(\tau_{1}\right) \in \mathrm{dy}\right\}=\mathrm{P}_{\mathrm{z}}\left\{\mathrm{T}_{\mathrm{N}_{1}} \in \mathrm{du} ; \int_{0}^{\mathrm{f}} \mathrm{f}(\mathrm{X}(\mathrm{t})) \mathrm{dt} \in \mathrm{dy}\right\}
\end{aligned}
$$

This completes the proof of the Theorem 4.1.

Note: From Theorem 3.1 and Theorem 4.1 we can derive many usefull and valuable information about the process $X(t)$ or additive functional $J_{f}(t)$. For this aim let's consider the following example.

\section{AN EXAMPLE}

Example 5.1. Using Theorem 3.1, let obtain the general form of the moments of the process $\mathrm{X}(\mathrm{t})$, when $\zeta_{\mathrm{i}}$ have the exponantial distribution with parameter $\mu>0$. From Theorem 3.1, it is known that

$$
\tilde{\mathrm{Q}}(\lambda, \mathrm{x}, \mathrm{z})=\tilde{\mathrm{G}}(\lambda, \mathrm{x}, \mathrm{z})+\mathrm{R}^{*}(\lambda, \mathrm{z}) \tilde{\mathrm{G}}(\lambda, \mathrm{x}, \bullet)\left[1-\mathrm{R}^{*}(\lambda, \bullet)\right]^{-1}
$$


If both sides of the formula (5.1) are multiplied by $\mathrm{e}^{-\mu z}$ and integrated respect to parameter z, we get:

$$
\tilde{\tilde{Q}}(\lambda, x, \mu)=\tilde{\tilde{G}}(\lambda, x, \mu)+\tilde{R}^{*}(\lambda, \mu) \tilde{\mathrm{G}}(\lambda, \mathrm{x}, \bullet)\left[1-\mathrm{R}^{*}(\lambda, \bullet)\right]^{-1}
$$

Let's write the following equalities in the formula (5.2):

$$
\tilde{\mathrm{G}}(\lambda, \mathrm{x}, \bullet)=\mu \tilde{\tilde{\mathrm{G}}}(\lambda, \mathrm{x}, \mu) ; \mathrm{R}^{*}(\lambda, \bullet)=\mu \tilde{\mathrm{R}}^{*}(\lambda, \mu) .
$$

Then we get:

$$
\tilde{\tilde{Q}}(\lambda, x, \mu)=\tilde{\tilde{\mathrm{G}}}(\lambda, \mathrm{x}, \mu)\left[1-\mu \tilde{\mathrm{R}}^{*}(\lambda, \mu)\right]^{-1} .
$$

Now, let calculate respectively $\tilde{\tilde{G}}(\lambda, x, \mu)$ and $\tilde{R}^{*}(\lambda, \mu)$. From Theorem 3.1,

$$
\mathrm{G}(\mathrm{t}, \mathrm{x}, \mathrm{z})=\sum_{\mathrm{n}=0}^{\infty} \Delta \Phi_{\mathrm{n}}(\mathrm{t}) \mathrm{a}_{\mathrm{n}}(\mathrm{x}, \mathrm{z}) ; \mathrm{R}(\mathrm{t}, \mathrm{z})=\sum_{\mathrm{n}=1}^{\infty} \Phi_{\mathrm{n}}(\mathrm{t}) \mathrm{b}_{\mathrm{n}}(\mathrm{z}-\mathrm{s})
$$

If both sides of the formula (5.4) are multiplied by $\mathrm{e}^{-\lambda t} \mathrm{e}^{-\mu z}$ and integrated respect to parameters $\mathrm{t}, \mathrm{z}$, we get:

$$
\tilde{\tilde{G}}(\lambda, x, \mu)=\frac{1-\varphi_{\xi}(\lambda)}{1-\varphi_{\xi}(\lambda) \varphi_{\eta}(\mu)} \frac{1-\mathrm{e}^{-\mu \mathrm{x}}}{\lambda \mu}, \varphi(\lambda)=\mathrm{E}\left(\mathrm{e}^{-\lambda \xi}\right), \lambda>0 .
$$

Using the similar methods, we get

$$
\tilde{R}^{*}(\lambda, \mu)=\frac{\varphi_{\xi}(\lambda)}{\mu} \frac{1-\varphi_{\eta}(\mu)}{1-\varphi_{\xi}(\lambda) \varphi_{\eta}(\mu)}
$$

The equalities (5.5) and (5.6) are written in the (5.3), we get:

$$
\tilde{\tilde{Q}}(\lambda, x, \mu)=\frac{1-\mathrm{e}^{-\mu \mathrm{x}}}{\lambda \mu}
$$

If the equality (5.7) is applied The laplace transform respect to $t$ and both of sides of the equality (5.7) is multiplied $\mu$, we obtain:

$$
\mathrm{Q}(\mathrm{t}, \mathrm{x}, \bullet)=1-\mathrm{e}^{-\mu \mathrm{x}}, \mu>0, \mathrm{x} \geq 0 \text {, for every } \mathrm{t} .
$$

Finally, let's get the moments of the process $X(t)$ :

$$
E\left(X^{n}(t)\right)=\int_{0}^{\infty} x^{n} Q_{x}^{\prime}(t, x, \bullet) d x=\int_{0}^{\infty} x^{n} \mu e^{-\mu z} d x=\frac{n !}{\mu^{n}}, n=1,2,3, \ldots
$$


It is known that $X_{s}(t)=s+X(t)$. Then, we can write the moments of the process $X_{s}(t)$ as follows:

$$
\mathrm{E}\left(\mathrm{X}_{\mathrm{s}}(\mathrm{t})\right)^{\mathrm{n}}=\mathrm{E}(\mathrm{s}+\mathrm{X}(\mathrm{t}))^{\mathrm{n}}=\sum_{\mathrm{k}=1}^{\mathrm{n}}\left(\begin{array}{l}
\mathrm{n} \\
\mathrm{k}
\end{array}\right) \mathrm{s}^{\mathrm{n}-\mathrm{k}} \mathrm{E}(\mathrm{X}(\mathrm{t}))^{\mathrm{k}}=\sum_{\mathrm{k}=1}^{\mathrm{n}}\left(\begin{array}{l}
\mathrm{n} \\
\mathrm{k}
\end{array}\right) \mathrm{s}^{\mathrm{n}-\mathrm{k}} \frac{\mathrm{k} !}{\mu^{\mathrm{k}}}, \mathrm{n}=1,2,3, \ldots
$$

Corollary 5.2. Using Theorem 3.1 , when $\zeta_{\mathrm{i}}$ have the exponantial distribution with parameter $\mu>0$, the the first four moments of the process $X_{s}(t)$ can be given as follows:
1) $E\left(X_{s}(t)\right)=s+\frac{1}{\mu}$;
2) $E\left(X_{s}^{2}(t)\right)=s^{2}+\frac{2 s}{\mu}+\frac{2}{\mu^{2}}$;
3) $E\left(X_{s}^{3}(t)\right)=s^{3}+\frac{3 s^{2}}{\mu}+\frac{6 s}{\mu^{2}}+\frac{6}{\mu^{3}}$;
4) $E\left(X_{s}^{4}(t)\right)=s^{4}+\frac{4 s^{3}}{\mu}+\frac{12 s^{2}}{\mu^{2}}+\frac{24 s}{\mu^{3}}+\frac{24}{\mu^{4}}$.

Corollary 5.3. Then, the variance of the process $X_{s}(t)$ can expressed as follows:

$$
\operatorname{Var}\left(X_{s}(t)\right)=E\left(X_{s}^{2}(t)\right)-E\left(X_{s}(t)\right)^{2}=\frac{1}{\mu^{2}}, \mu>0
$$

Corollary 5.4. Let be $M_{k}=E\left[X_{s}(t)-E\left(X_{s}(t)\right)\right]^{k}, k=1,2,3,4$. The first four central moments of the process $\mathrm{X}_{\mathrm{s}}(\mathrm{t})$ can be written as follows:
1) $\mathrm{M}_{1}=0$;
2) $\mathrm{M}_{2}=\frac{1}{\mu}$
3) $\mathrm{M}_{3}=\frac{2}{\mu^{3}}$
4) $\mathrm{M}_{4}=\frac{9}{\mu^{4}}, \quad \mu>0$.

Corollary 5.5. The asymmetry-symmetry coefficients of the process $X_{s}(t)$ can be written as follows:
1) $\gamma_{3}=\frac{M_{3}}{\left(\sqrt{M_{2}}\right)^{3}}=2$;
2) $\gamma_{4}=\frac{M_{4}}{M_{2}^{2}}-3=6$

Conclusion. Using similar methods, from Theorem 3.1 and Theorem 4.1 the different corollaries, very important for solving some problems of applied sciences,.can be obtained. 
Acknowledgement: We would like to express our regards to Professor A.V. Skorohod, Michigan State University, for his support and encouragement which led us to do the further investigations on the processes with a discrete interference of chance and its applications. Moreover, Assoc. Prof. Rovshan Aliyev would like to express his thanks to TUBITAK for inviting him to Karadeniz Technical University, Faculty of Art and Sciences, Department of Statistics and Computer Sciences and awarding a "Fellowship for visiting scientist" scholarship.

\section{REFERENCES}

1. G. Alsmeyer, Some relations between harmonic renewal measures and certain first passage times, Statistics and Probability Letters, 12, 1, 19-27, 1991.

2. M. Brown and H. Solomon, A second-order approximation for variance of a renewal reward process, Stochastic Processes and Their Application, 3, 301-314, 1975.

3. W. Feller, An Introduction to Probability Theory and Its Applications II., 2nd Ed. New York, 1971.

4. W. S. Jewell, Fluctuation of a Renewal- Reward Process, Journal of Mathematical Analysis and Applications, 19, 309-329, 1967.

5. T. A. Khaniyev and I. Unver, The study of the level zero crossing time of a semiMarkovian random walk with delaying screen, Turkish Journal of Mathematics, 21, 3, 257-268, 1997.

6. T. A. Khaniyev, Some asymptotics results for the semi-Markovian random walk with a special barrier, Turkish Journal of Mathematics, 27, 2, 257-268, 2003.

7. T. A. Khaniev and Z. Kucuk, Asymtotic expansions for the moments of the Gaussian random walk with two barriers, Statistics and Probability Letters, 69, 1, 91-103, 2004.

8. T.A. Khaniyev, T. Kesemen, O. Kesemen and R.T. Aliyev, Some asymptotic results for the stationary characteristics of the semi-markovian random walk with barrier, Automatic Control and Computer Sciences, 1, 31-43, 2006.

9. T.A. Khaniyev and Z. Mammadova, On the stationary characteristics of the extended model of type $(\mathrm{s}, \mathrm{S})$ with Gaussian distribution of summands, Journal of Statistical Computation and Simulation, 76, 10, 861-874, 2006.

10. T. A. Khaniev, About moments of generalized renewal process, Transactions of NAS of Azerbaijan, Series of Phy. Tech. and Math. Sciences, 25, 1, 95-100, 2005.

11. V. I. Lotov, On some boundary crossing problems for Gaussian random walks, The Annals of Probability, 24, 4, 2154-2171, 1996. 